\title{
Machine vision technology for agricultural applications ${ }^{\text {2 }}$
}

\author{
Yud-Ren Chen*, Kuanglin Chao, Moon S. Kim \\ Instrumentation and Sensing Laboratory, Henry A. Wallace Beltsville Agricultural Research Center, \\ Agricultural Research Service, US Department of Agriculture, Building 303, 10300 Baltimore Ave, Beltsville, \\ MD 20705-2350, USA
}

\begin{abstract}
Current applications of machine vision in agriculture are briefly reviewed. The requirements and recent developments of hardware and software for machine vision systems are discussed, with emphases on multispectral and hyperspectral imaging for modern food inspection. Examples of applications for detection of disease, defects, and contamination on poultry carcasses and apples are also given. Future trends of machine vision technology applications are discussed.
\end{abstract}

(C) 2002 Elsevier Science B.V. All rights reserved.

Keywords: Multispectral; Hyperspectral; Quality; Safety; Inspection; Real-time

\section{Introduction}

Many applications using machine vision technology have been developed in agricultural sectors, such as land-based and aerial-based remote sensing for natural resources assessments, precision farming, postharvest product quality and safety detection, classification and sorting, and process automation. This is because machine vision systems not only recognize size, shape, color, and texture of objects, but also provide numerical attributes of the objects or scene being imaged.

\footnotetext{
Company and product names mentioned are used for clarity only and do not imply any endorsement by USDA to the exclusion of other comparable products.

* Corresponding author. Tel.: 301-504-8450; fax: +301-504-9466

E-mail address: cheny@ba.ars.usda.gov (Y.-R. Chen).
} 
Besides imaging objects in the visible (VIS) color region, some machine vision systems are also able to inspect these objects in light invisible to humans, such as ultraviolet (UV), near-infrared (NIR), and infrared (IR). The information received from objects in invisible light regions can be very useful in determining preharvest plant maturity, disease, or stress states. It is very useful in determining plant and vegetable variety, maturity, ripeness, and quality. It is also useful in detecting postharvest quality and safety, such as defects, composition, functional properties, diseases and contamination of plants, grains and nuts, vegetables and fruits, and animal products.

Advantages of using imaging technology for sensing are that it can be fairly accurate, nondestructive, and yields consistent results. Applications of machine vision technology will improve industry's productivity, thereby reducing costs and making agricultural operations and processing safer for farmers and processing-line workers. It will also help to provide better quality and safe foods to consumers.

Machine vision discussed here is limited to camera machine vision systems. It holds great potential and benefits for the agricultural industry because of its simplicity, low cost, rapid inspection rate, and broad range of applications. Machine vision can also be performed using X-ray imaging and nuclear magnetic resonant imaging (MRI). X-ray and MRI imaging are widely used in medical applications. Even though they have potential for detecting diseases and defects in agricultural products and food (Chen et al., 1989; Schatzki et al., 1997; Marks et al., 1998), their applications in the agricultural sector are limited because of the high cost of equipment investment and low operational speed.

\section{Components of a machine vision system}

Machine vision systems commonly used in agricultural applications acquire reflectance, transmittance, or fluorescence images of the agricultural materials under UV, VIS, or NIR illumination. A basic machine vision system consists of a camera, a computer equipped with an image acquisition board, and a lighting system. Also, computer software is required for transmitting electronic signals to computers, acquiring images, and performing storage and processing of the images.

\subsection{Lighting}

The light range can be in the UV (200-400 nm), VIS (400-700 nm), or NIR (700$2500 \mathrm{~nm}$ ). There are also applications in thermal imaging (above $2500 \mathrm{~nm}$ ) for agricultural products. When radiation from the lighting system illuminates an object, it is transmitted through, reflected, or absorbed. These phenomena are referred to as optical properties. The absorbed light can also be re-emitted (fluorescence), usually at longer wavelengths. A number of compounds emit fluorescence in the VIS region of the spectrum when excited with UV radiation. The optical properties and fluorescence emission from the object are integrated functions of the angle and wavelength of the incident light and chemical and physical composition of the object. 
The importance of proper illumination for a machine vision system cannot be overstated. With a well-chosen lighting system, the incident light will present the objects or scenes in the optimal way to be recognized or analyzed, thereby eliminating many tedious image processing procedures that otherwise would be needed. The lighting unit selection and its configuration in a machine vision system depend on the application. At present, lighting hardware is readily available for common machine vision applications in agriculture.

\subsection{Frame grabber}

Many monochrome frame-grabber boards are capable of taking RS-170 or CCIR video inputs, while the color frame-grabber receives NTSC, PAL, or S-VHS input signals. The features of a frame grabber required for machine vision applications include image acquisition, camera control, and image data pre-processing. The frame grabber can acquire either digital or analog images depending on the camera used. For camera control, a minimum requirement is accurate A/D circuitry and precise camera timing. Input signal conditioning, such as the ability to control gain and offset, is important to minimize effects from camera variability or lighting fluctuations. Also, some frame grabber boards are capable of preprocessing imaging with functions such as "first-in-first-out" (FIFO) and "look-up table" (LUT).

A modern frame grabber board can communicate with the host CPU's memory via software driver at speeds of $80-130$ Mbytes/s (PCI-bus interface). This speed is enough to meet the needs of many real-time operation for agricultural applications.

\subsection{Image processing and analysis software}

Digital image processing is performed with a computer to manipulate information within an image to make it useful. Image processing in agricultural applications may consist of three steps: (1) image enhancement, (2) image feature extraction, and (3) image feature classification. Image enhancement is commonly applied to a digital image to correct problems such as poor contrast or noise. Image enhancement procedures such as morphological operations, filters, and pixel-to-pixel operations are generally used to correct inconsistencies in the acquired images caused by inadequate and/or nonuniform illumination. Statistical procedures from basic image statistics such as mean, standard deviation, and variance to more complex measurement such as principle component analysis can be used to extract features from digital images. Once image features are identified, the next step is feature classification. Numerical techniques such as neural networks and fuzzy inference systems can be successfully applied to perform image feature classification.

\subsection{CCD cameras}

Machine vision systems utilize imaging cameras ranging from monochrome cameras performing simple shape and size recognition tasks to common aperture 
multispectral cameras for detection of surface defects and diseases on meat, grains, fruits, and vegetables.

An imaging camera receives light from the object surface and converts the light into electrical signals using a charge-coupled device (CCD). CCDs are solid state, silicon-based devices and are available in either linear or area array configurations. Linear array CCD sensors are able to sense a line of pixels during a single exposure. It is used to capture a full two-dimensional object image through motion of either the object or the sensor along the direction perpendicular to the line of pixels. Area arrays are able to capture a two dimensional image with a single exposure, but are much more expensive to manufacture, especially in the larger array sizes. A light sensitive CCD device converts an optical image into an array of electrical signals. The electrical signals are proportional to the intensity of the light from the surface. An A/D device converts the electrical signals into an 8- or 16-bit data, and the digitized imaging data are then stored in the computer.

\subsubsection{Monochrome imaging}

Monochrome imaging requires a single-chip CCD. It is able to sense VIS and NIR if it is so designed.

The resolution of a CCD image depends on how many pixels are in the CCD arrays. Depending on the nature of applications, the camera resolution can range from 480 to 1024 lines or even higher.

Various monochrome imaging techniques have been used for the determination of agricultural product quality. For example, monochrome machine vision techniques were used for automatic segmentation of the rib-eye area from a cut surface of longissimus muscle and for the determination of the degree of marbling in the beef rib-eye area (McDonald and Chen, 1991, 1992; Hwang et al., 1997). It was also used for the detection of blemishes and bruises on apples (Davenel et al., 1988; Rehkugler and Throop, 1989; Singh and Delwiche, 1994; Throop et al., 1995). Monochrome machine vision technology was also used for detecting scars, cracks, and spreading tips for asparagus (Rigney et al., 1996). Grading apples with on-line machine vision has been attempted (Rehkugler and Throop, 1989; Throop et al., 1995). The major challenges for on-line inspection are to produce quality images that provide clearly identifiable features and to have both efficient hardware and software to process the images fast enough for on-line implementation.

\subsubsection{Color imaging}

A single chip CCD camera can also be used for color imaging. This is done by alternating the pixels in the CCD camera for red, green, and blue (RGB) color acquisition in the area array CCD to simulate the colors seen by the human eye. However, this technique, which is adequate for television or video viewing, may not be suitable for complicated machine vision applications.

Color imaging can also be achieved by using three-chip CCD camera systems. Each CCD in a three-chip camera receives RGB colors to produce near true color images of the objects. This is accomplished by using a prism assembly with bandpass filters and a dichroic coating on selected surfaces of the prisms that separate broad 


\section{Green}

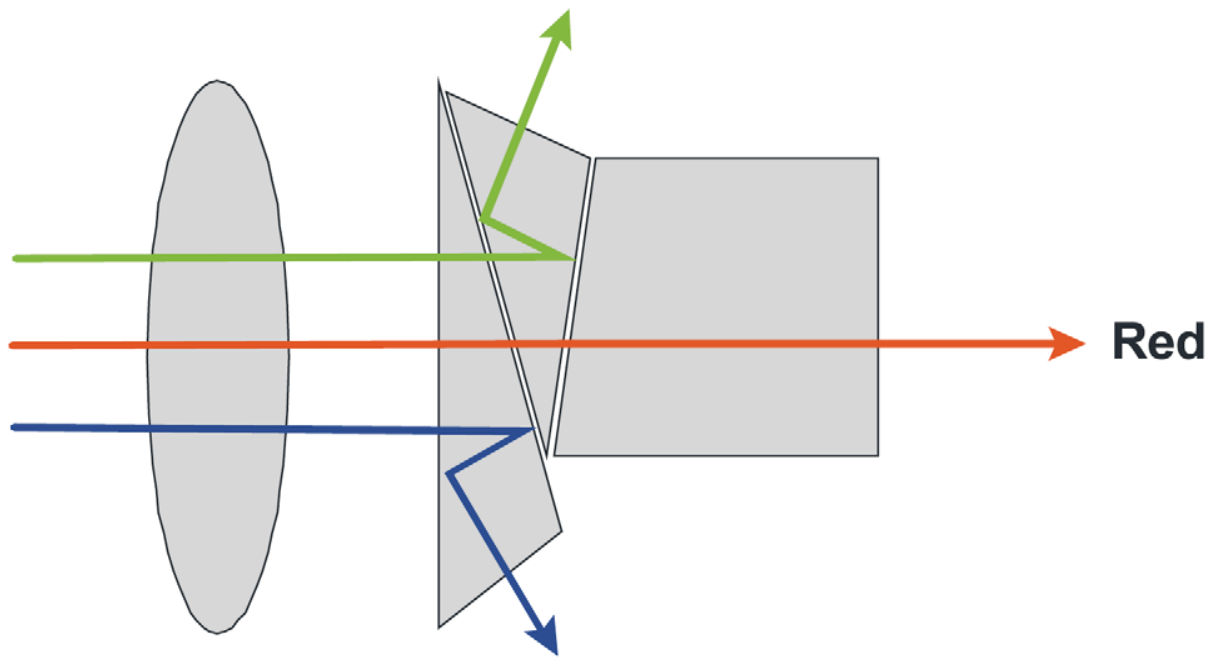

Blue

Fig. 1

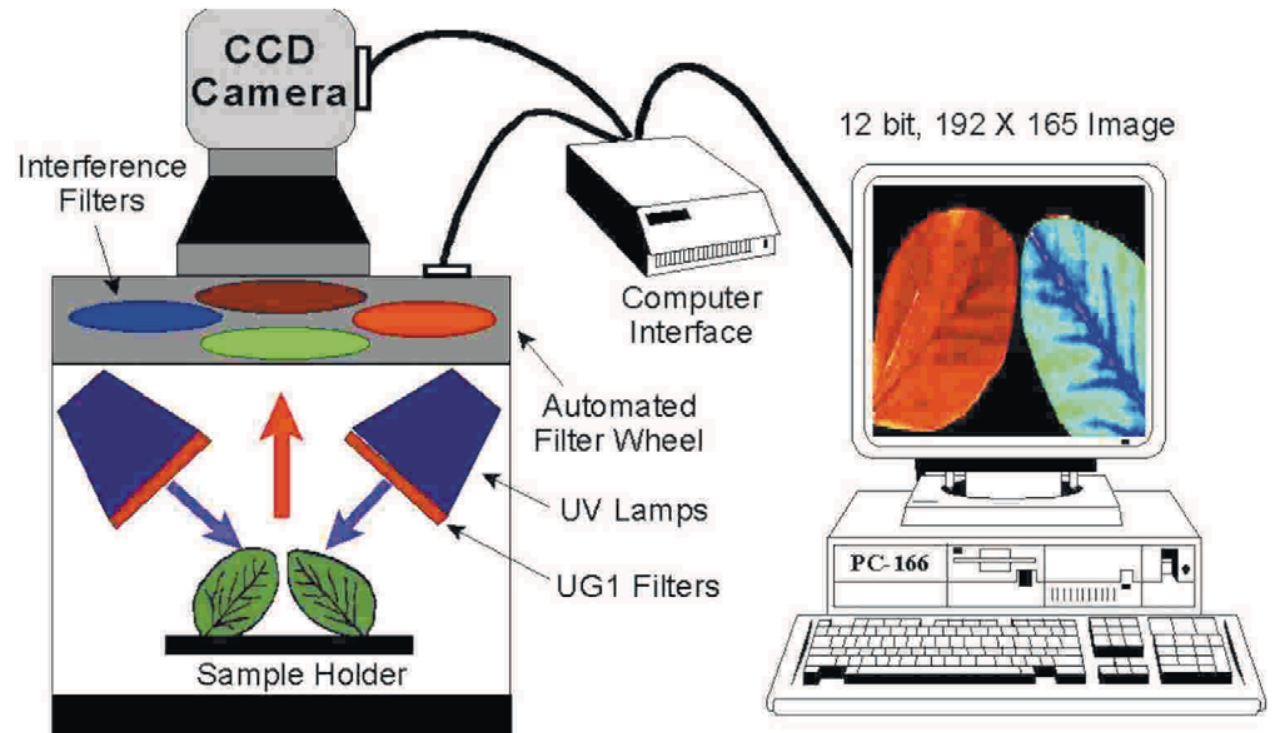

Fig. 2

Fig. 1. Three-chip color imager.

Fig. 2. Multispectral imaging system with a rotating filter wheel. 
band light into RGB channels. The image acquired by each CCD is monochromatic either for red, green, or blue (Fig. 1). Hence, a composition of the three-channel signals provides a near true color image of the object.

There are many applications in color imaging for detection of agricultural product quality. Throop et al. (1993) used a color difference between bruised and nonbruised regions on 'Golden Delicious' apples. Daley et al. $(1993,1995)$ applied color imaging techniques to on-line poultry quality grading. A color imaging system was used at the Instrumentation and Sensing Laboratory (ISL) to classify livers and hearts of wholesome and unwholesome chickens (Chao et al., 1999). The unwholesome chickens had syndromes of airsacculitis, cadaver, and septicemia/toxemia. The accuracy for separation of livers from wholesome and unwholesome chickens were found to range from 87.5 to $92.5 \%$, and hearts, from 92.5 to $97.5 \%$.

\subsubsection{Multispectral imaging}

Multispectral imaging consists of a set of several images, each acquired at a narrow band of wavelengths. The simplest method to obtain images at a discrete spectral region is by positioning a bandpass filter (or interference filter) in front of a monochrome camera lens. Multispectral images can be obtained by capturing a series of spectral images by using either a liquid crystal tunable filter (LCTF) or an acousto-optic tunable filter, or by sequentially changing filters in front of the camera. Fig. 2 shows a multispectral imaging system with a rotating filter wheel (Kim et al., 2001a) mounted with four filters for imaging fluorescence emission of plant leaves.

A more advanced approach in multispectral imaging is the use of a commonaperture multi-channel imaging camera. A three-channel common-aperture multispectral imaging camera is similar to the three-chip color camera. The range of spectral regions are accomplished by proper selections of dichroic coatings and bandpass filters. With the same principle, a two-, four-, or six-channel common aperture camera can also be built. The advantage of common aperture multispectral imaging is that it can simultaneously acquire multiple spectral images. This can facilitate high-speed acquisition and accurate processing, such as subtractions and additions, of multiple images of different spectral bands.

Taylor and McCure (1989) used a multispectral imaging system, with a rotating wheel holding six optical filters. They demonstrated that three wavelengths, 670, 800, and $990 \mathrm{~nm}$, could detect healthy and unhealthy leaf tissues. They also demonstrated that it could map chlorophyll distribution over the leaf surface. Muir et al. (1982) used spatial information at eight wavelengths to detect $12-15$ kinds of blemishes on a potato. At ISL, Park and Chen (1994) used an intensified multispectral imaging system to discriminate wholesome poultry carcasses from unwholesome carcasses. Park and Chen (1996) reported the performance of a co-occurrence matrix textural analysis method as a tool of multispectral image analysis for detecting unwholesome poultry carcasses. Multispectral images were used to characterize chicken heart images for disease detection (Chao et al., 2001). Multispectral fluorescence imaging was shown to be useful in studying diffusion of herbicide within leaves, after they were treated with the herbicide (Kim et al., 2001a). 


\subsubsection{Hyperspectral imaging}

In recent years, hyperspectral imaging has emerged as a powerful technique in earth remote sensing and medical diagnosis. This technique combines the features of imaging and spectroscopy to acquire both spatial and spectral information from an object. The technique yields much more useful information than other imaging techniques, because each pixel on the image surface possesses a spectral signature of the object at that pixel.

Spectroscopic data analysis techniques can be used to extract chemical composition from each or an aggregate of pixels. Because of these combined features, hyperspectral imaging can greatly enhance our capability to identify materials and detect subtle and/or minor features in an object. Applications range from precision agriculture applications, such as detection of plant stress or crop infestation, to medical applications, and agricultural product quality and safety sensing.

Two general approaches have been used in the development of hyperspectral imaging techniques. One of the approaches is to sequentially capture a series of narrow-band spectral images to accomplish a three-dimensional image cube. Another approach is a pushbroom method where a line of spatial information with a full spectral range per spatial pixel is captured sequentially to complete a volume of spatial-spectral data. The fact that CCD detectors have two-dimensional arrays and a spectrograph allows simultaneous recording of a line of spatial and a multiple of spectral information. The advantage of this type of system is that sample sizes in one of the spatial directions (Fig. 3) are not limited by the size of CCD as compared to the first approach that sequentially captures a series of narrow-band spectral images.

Martinsen and Shaare (1998) applied hyperspectral imaging to measure soluble solids distribution in kiwifruit and found the technique very promising. Mao and Heitschmidt (1999) reported a hyperspectral imaging system with the capability of both airborne and ground/laboratory data acquisitions. They used a LCTF, a CCD video camera, a frame grabber, and a portable computer system. The spectral range is from 450 to $750 \mathrm{~nm}$ with a $10 \mathrm{~nm}$ bandpass. The system is able to capture different spectral images at up to 14 images per second.

\section{Case studies}

\subsection{On-line poultry inspection by a multi-camera system}

There is an urgent need to develop automated inspection systems that can operate on-line in real-time (at least 140 birds per minute) in the poultry slaughter plant environment. These systems should be able to accurately detect and identify carcasses unfit for human consumption.

Based on an early study (Park et al., 1998) using industrial machine frames, a transportable dual-camera system for separating wholesome and unwholesome chicken carcasses on-line was assembled (Chao et al., 2000). The dual cameras were equipped with filters with center wavelengths at 542 and $700 \mathrm{~nm}$, respectively. A 

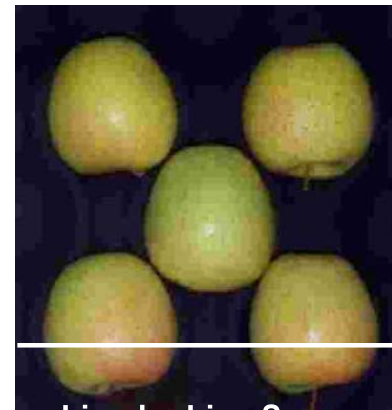

\section{Line-by-Line Scan}

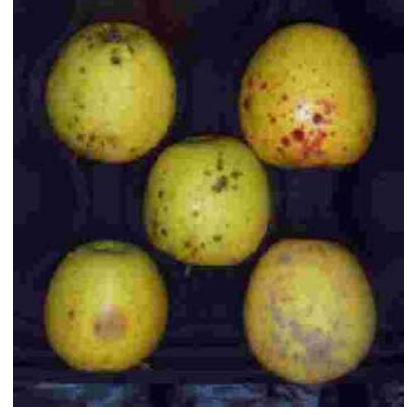

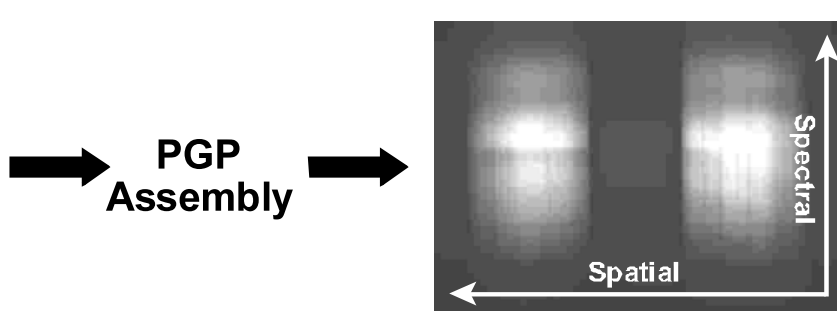

CCD

Fig. 3. Line scan of apples with a PGP assembly imager.

schematic of the dual-camera system is shown in Fig. 4. The description of its major components is given in Chao et al. (2000), where a laboratory version of the system is described. In the transportable system, two fiber-optic dual-line lights equipped with AC-regulated $150 \mathrm{~W}$ quartz-halogen light bulbs were used to provide evenly distributed illumination to the poultry carcasses. The dual-line lights were positioned bilaterally at $45^{\circ}$ angles to provide balanced area illumination to the poultry carcass.

For this machine vision inspection system, object-oriented programming paradigms (Rumbaugh et al., 1991) were utilized to integrate the hardware components. The image is reduced to a size of $256 \times 240$ pixels and then the carcass is segmented from the background using simple thresholding. A total of 15 horizontal layers (16 horizontal lines of pixels each) are generated from each segmented image, as shown in Fig. 5. For each layer, a centroid is calculated from the binarized image. Based on these centroids, each layer was divided into several square blocks $(16 \times 16$ pixels $)$, for a total of 107 blocks. The averaged intensity of each block is used as the input data to neural network models. The constant number of blocks in each layer was previously determined to delineate the main part of each carcass and omit the legs and wings. For a very small chicken, the edge blocks could contain several background pixels, passing chicken size information on to the neural net in the form of lowered average intensity. 

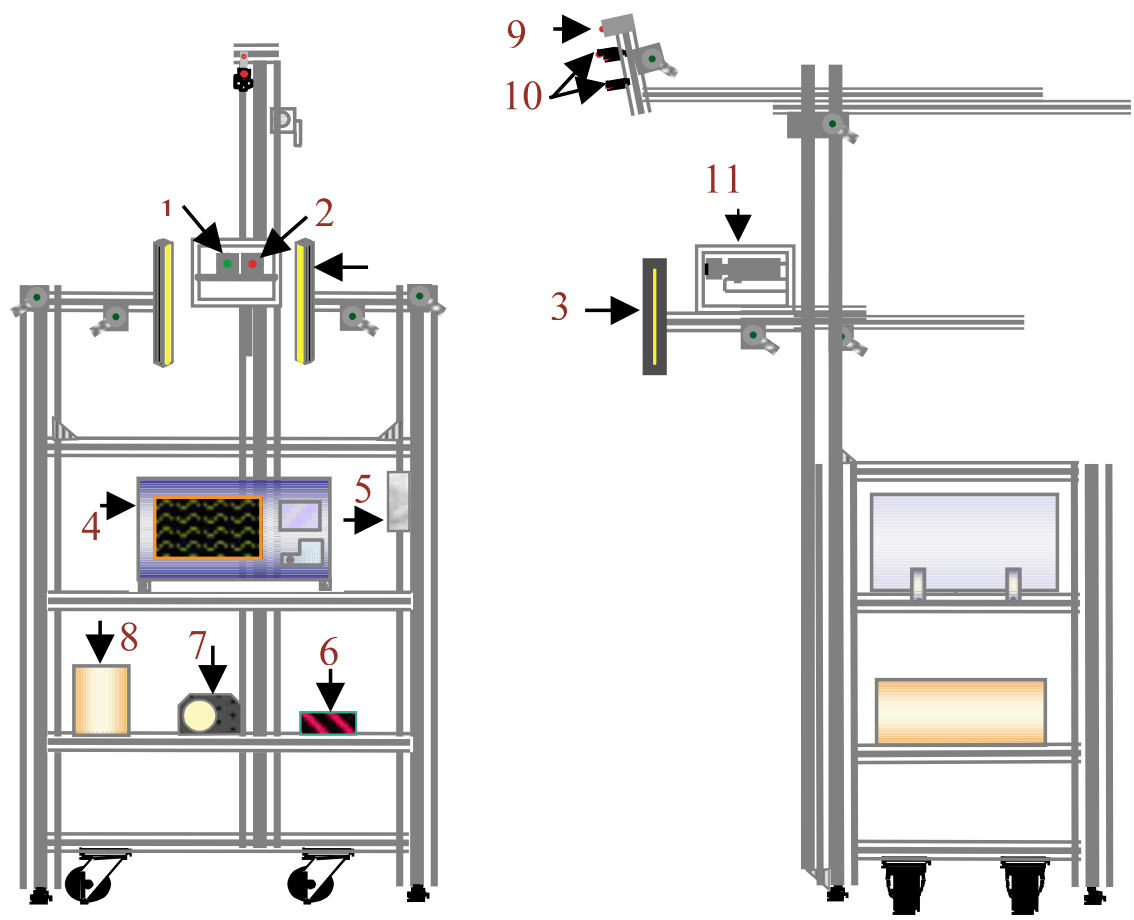

Fig. 4. Schematic of transportable dual-camera inspection system: (1) camera w/540 nm filter, (2) camera w/700 nm filter, (3) fiber optic dual-line illuminator, (4) industrial computer, (5) interface and camera control box, (6) $12 \mathrm{~V}$ power supply to the dual-camera, (7) fiber optic light source, (8) battery backup (UPS), (9) photoelectric proximity sensors, (10) magnetic proximity sensor, (11) camera enclosure.

A backpropagation neural network model for classification was done off-line from images acquired on-line. These parameters are then incorporated into the on-line classification section of the software. The feed-forward-back-propagation neural network model has 107 input nodes, 10 nodes in one hidden layer, and 2 output nodes. The output nodes' target outputs are $\left(\begin{array}{ll}0 & 1\end{array}\right)$ or $(10)$, depending on whether the sample was identified wholesome or unwholesome by the veterinarian. For each of the three data sets, model development method starts with splitting the data into two sub-sets: training (50\%) and validation (50\%). Each sub-set contains equal numbers of wholesome and unwholesome carcasses. The neural network models are trained on the training sub-set. The validation sub-set is used to decide which network model and how much training is optimal. Training is always stopped after 15000 iterations.

Fig. 6 shows typical images for sampled poultry carcasses at two wavelengths. Typical images of wholesome carcasses and three kinds of unwholesome carcasses (septicemia, cadaver, and airsacculitis) are shown. The reflectance intensity of wholesome carcasses was not sensitive to the wavelength filters. As shown in (g) and (h), little difference existed in reflectance intensity between wavelengths at 500 and 
(a)

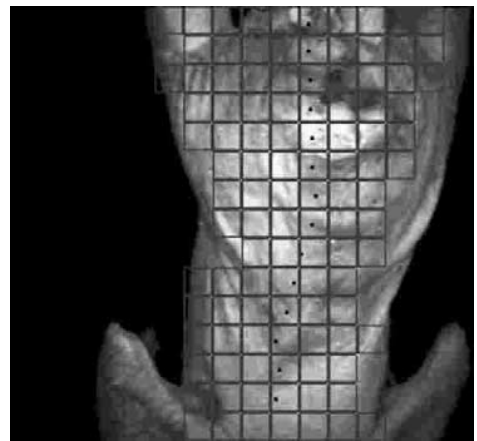

(c)

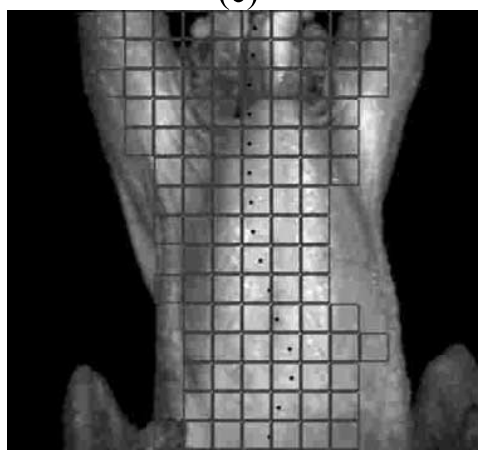

(b)

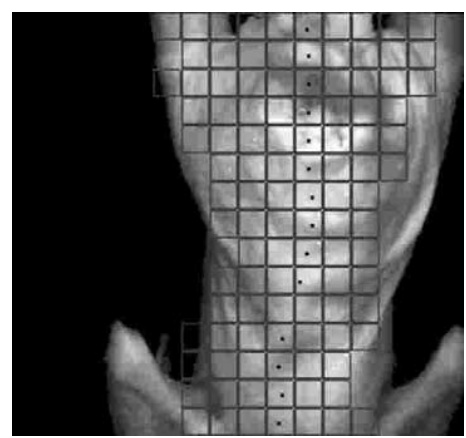

(d)

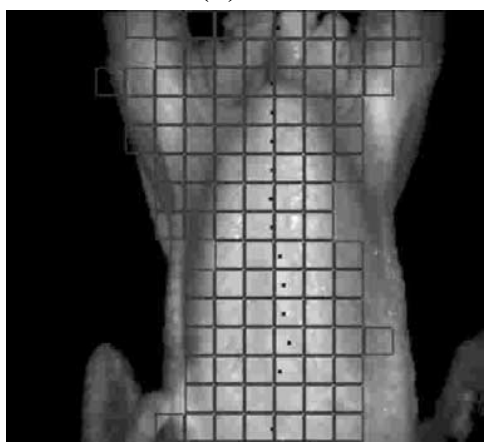

Fig. 5. Real-time image processing from the MVIS. Centroid and mesh generation during image capture for off-line training. (a) front at $540 \mathrm{~nm}$, (b) front at $700 \mathrm{~nm}$, (c) back at $540 \mathrm{~nm}$, (d) back at $700 \mathrm{~nm}$.

Table 1

Number of carcasses used for model development and on-line testing

\begin{tabular}{lcc}
\hline Date collected & Wholesome & Unwholesome \\
\hline Model development & & \\
9/16/99-9/20/99 & 500 & 500 \\
9/21/99-9/22/99 & 150 & 150 \\
9/27/99 & 50 & 50 \\
Total & 700 & 700 \\
On-line testing & 5952 & 395 \\
9/28/99-9/30/99 & 5952 & 395 \\
Total & & \\
\hline
\end{tabular}

$700 \mathrm{~nm}$. However, the reflectance intensities for unwholesome carcass at 540 and 700 $\mathrm{nm}$ were significantly different from that of wholesome carcasses. For unwholesome chicken carcasses, the reflectance with the filter of the $540 \mathrm{~nm}$ wavelength was darker than the intensity with a $700 \mathrm{~nm}$ filter $(\mathrm{a}-\mathrm{f})$. This shows that the unwholesome 


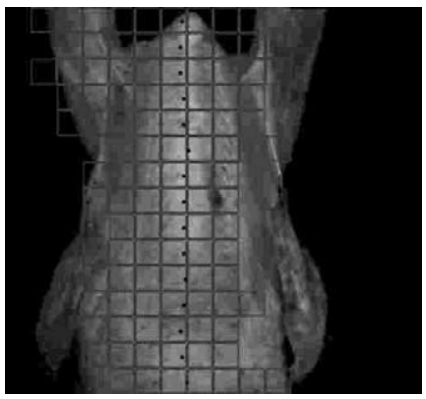

(a) air-sac $@, 540 \mathrm{~nm}$

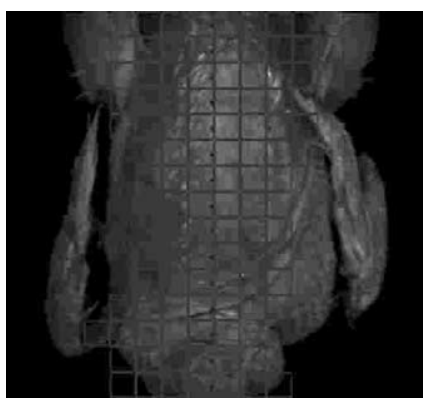

(c) cadaver $(a, 540 \mathrm{~nm}$

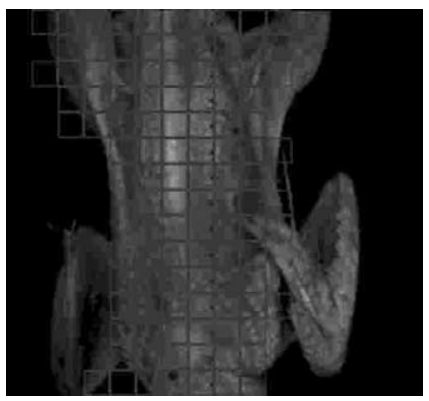

(e) septicemia $@, 540 \mathrm{~nm}$

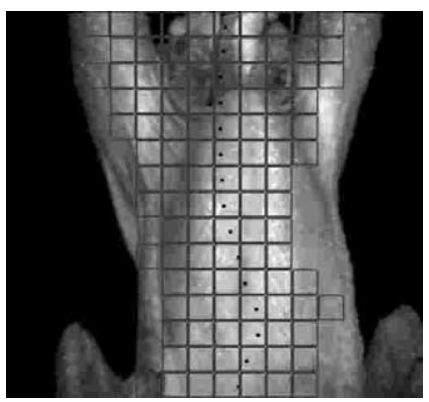

(g) normal $@, 540$ nm

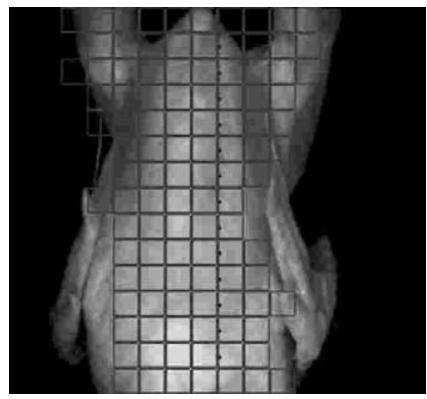

(b) air-sac $@, 700$ nm

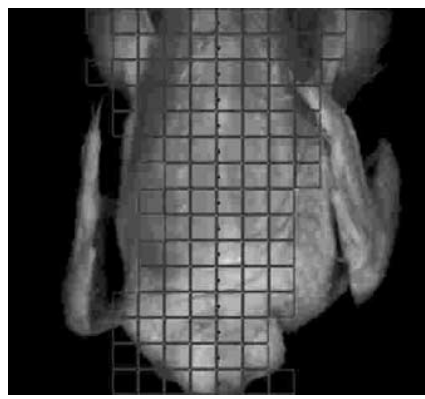

(d) cadaver $(a, 700 \mathrm{~nm}$

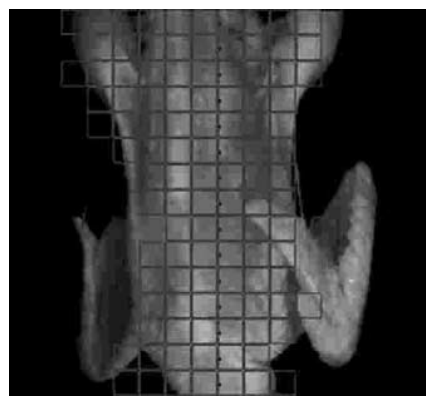

(f) septicemia $(a, 700 \mathrm{~nm}$

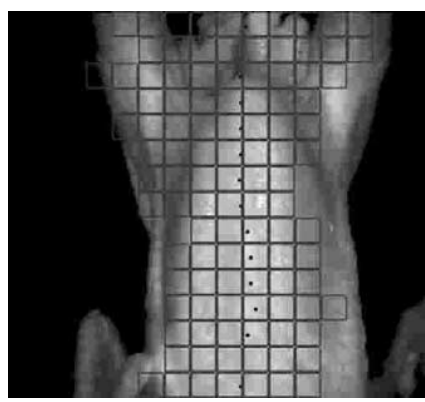

(h) normal $@, 700 \mathrm{~nm}$

Fig. 6. Real-time multi-spectral images for poultry carcass inspection. 


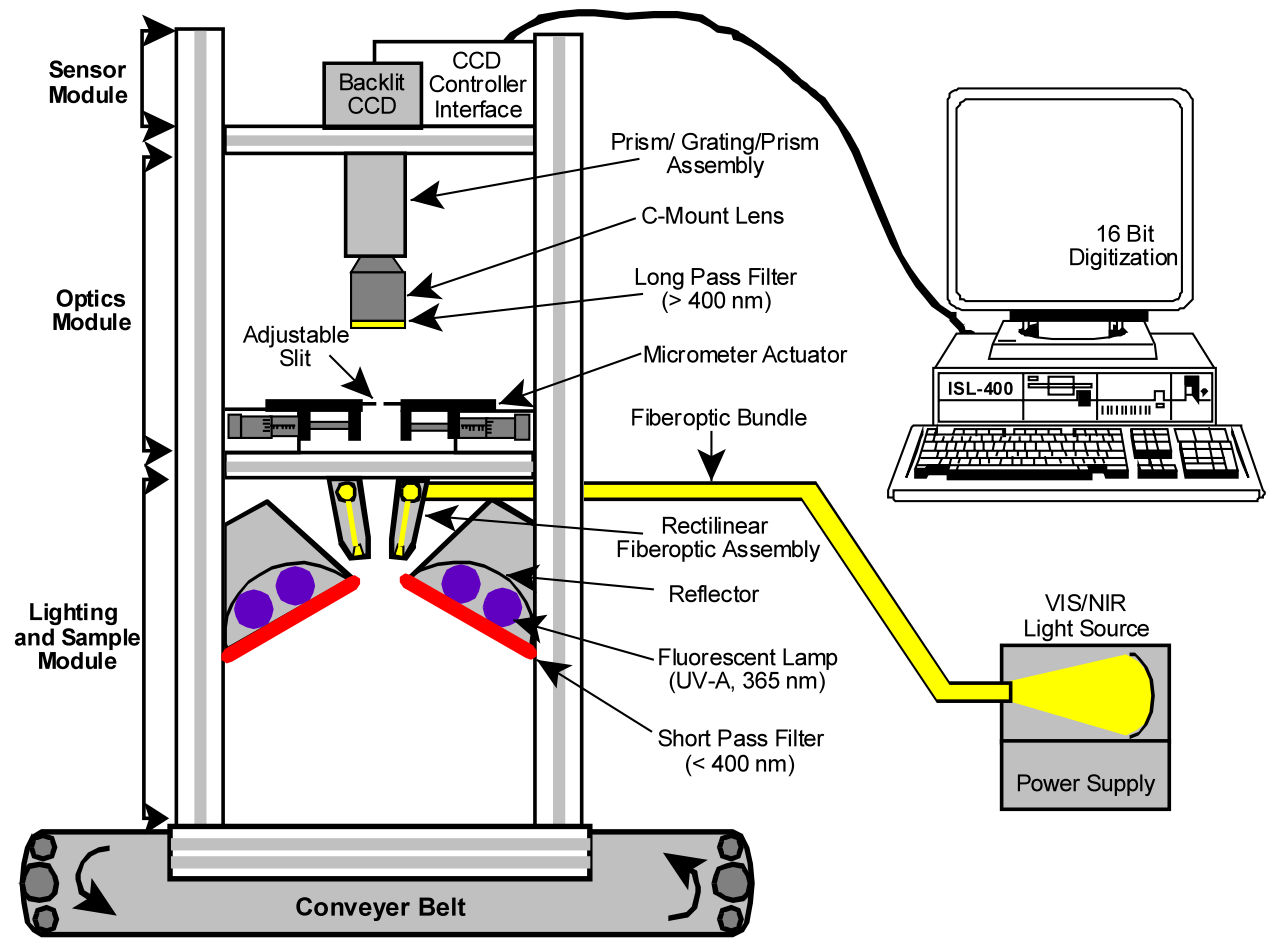

Fig. 7. ISL hyperspectral imaging system for food safety study.

spectral images at the $700 \mathrm{~nm}$ wavelength were not the same as those at the $540 \mathrm{~nm}$ wavelength. Thus, the combination of these two wavelengths enabled the differentiation of wholesome carcasses from unwholesome carcasses.

The dual-camera system was installed between the evisceration station and inspector station. A total of 1400 poultry carcasses (700 wholesome and 700 unwholesome) were measured for development of classification models. It was used to test a total of 6347 poultry carcasses (5952 wholesome and 395 unwholesome) online (Table 1). In each case, the 540 and $700 \mathrm{~nm}$ results were combined using an

Table 2

Classification accuracy for on-line testing

\begin{tabular}{|c|c|c|c|c|c|}
\hline \multirow[t]{2}{*}{ Test on day(s) } & & & \multicolumn{3}{|l|}{ Predicted } \\
\hline & & & Wholesome & Unwholesome & Accuracy $(\%)$ \\
\hline \multirow[t]{2}{*}{ 9/28/99-9/30/99 } & \multirow[t]{2}{*}{ Actual } & Wholesome & 5599 & 353 & 94.0 \\
\hline & & Unwholesome & 50 & 345 & 87.3 \\
\hline
\end{tabular}


AND operation to give a single prediction. That is, a carcass is predicted wholesome only if the data from both cameras result in wholesome prediction. Table 2 gives the results of the on-line testing. Of a total of 5952 wholesome carcasses, 5599 carcasses were predicted correctly (94\%), and $87 \%$ of the 395 unwholesome carcasses were correctly predicted.

3.2. Detection of apple diseases, defects, and contamination by hyperspectral imaging system

Applications of hyperspectral imaging technology to inspection and grading of food and agricultural products for quality and safety at ISL started in 1998. A preliminary study on identifying normal and abnormal poultry carcasses using hyperspectral imaging was conducted by Lu and Chen (1998). Since then, the ISL hyperspectral imaging system was redesigned so that it can be used to evaluate reflectance and fluorescence images (spectral range from 425 to $950 \mathrm{~nm}$ ) of agricultural products, with very high spatial and spectral resolutions (Kim et al., 2000, 2001b).

Fig. 7 shows the schematic diagram and hardware components of the ISL hyperspectral imaging system. The sensor module includes a back illuminated CCD and a control unit (Pixel Vision, Inc., Tigard, Oregon) that interfaces with a Pentium-based personal computer. The CCD has $512 \times 512$ pixel elements with a 16bit dynamic data range and is thermo-electrically cooled.

A spectrograph (ImSpector-V9, Spectral Imaging Ltd., Oulu, Finland) coupled with a $\mathrm{C}$-mount lens is attached to the CCD camera head. The spectrograph consists of a prism-grating-prism (PGP) construction that is a holographic transmission

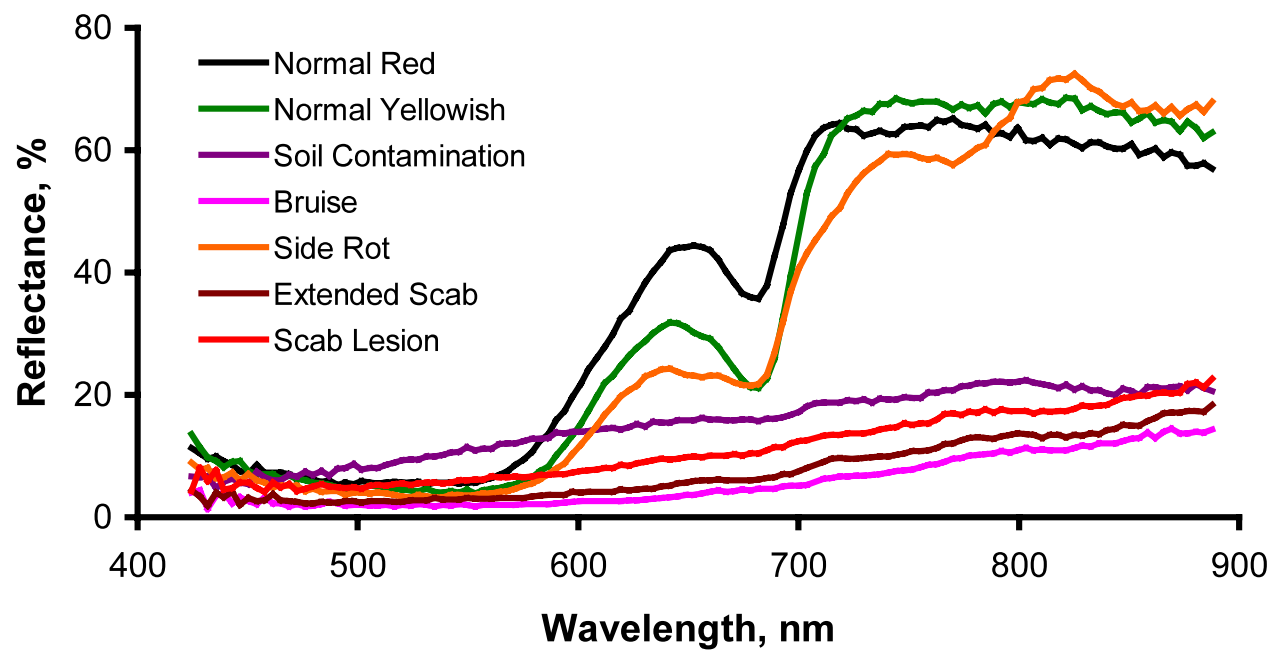

Fig. 8. Comparison of reflectance spectra for Red Delicious apples. 
grating. This assembly disperses incoming light into a spectral and spatial matrix, which then impinges onto the CCD.

Two independent light sources for the reflectance and fluorescence imaging are incorporated into the system. Sample illumination for reflectance measurements is provided by two $150 \mathrm{~W}$ halogen lamps powered by two regulated DC power supplies. The light is collected and transmitted through two rectilinear fiber bundles, which provide near uniform illumination of samples.

For fluorescence measurements, two UV-A fluorescent lamp assemblies are arranged to provide near uniform excitation energy to the sample area. Low-pass filters are placed in front of the lamp housing to prevent transmittance of radiation greater than approximately $400 \mathrm{~nm}$, therefore eliminating spectral contamination by pseudo-fluorescence.

Following is an example of an application of hyperspectral reflectance images for detection of contaminated Red Delicious apples. Because of the highly nonuniform surface color of the Red Delicious apples, detection of contaminations on these apples, among all cultivars of apples, presents a challenging task for machine vision applications.

The contaminations studied at ISL included physical damages such as bruises, side rot, scabs, and soil contamination. The normal or uncontaminated apple portions included those of reddish and yellow-greenish colors.

Fig. 8 illustrates typical spectra extracted from the hyperspectral image data for the uncontaminated and contaminated surfaces of the apples. In general, unconta-

(a)

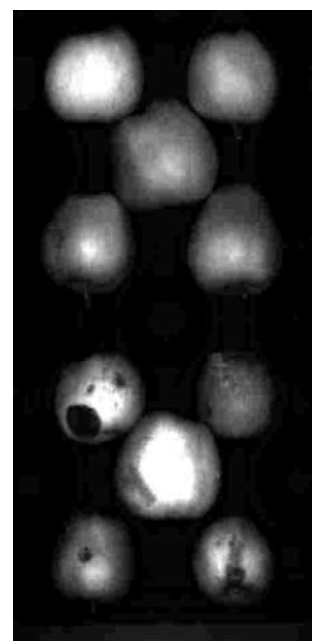

(b)

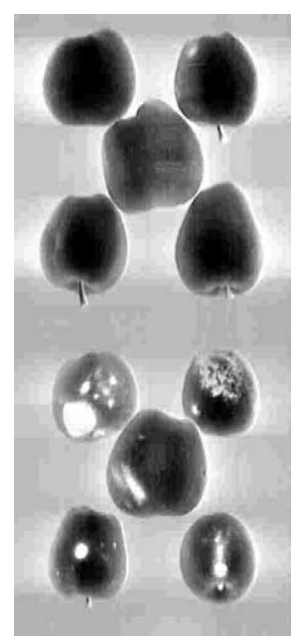

(c)

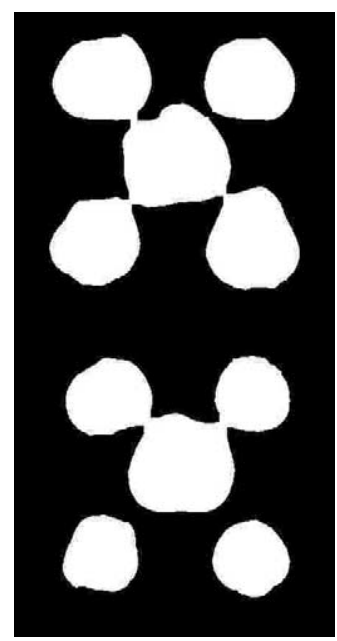

(d)

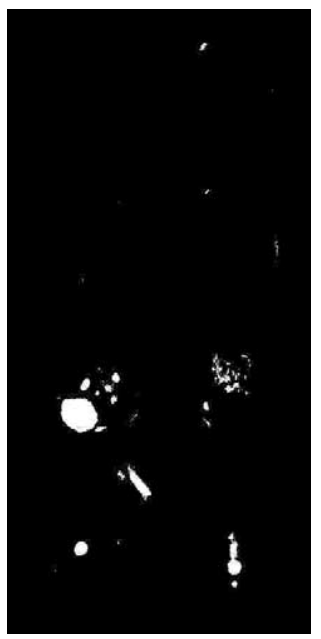

Fig. 9. (a-d) Simple image processing procedure on apples: (a) images at the chlorophyll absorption band, (b) images after applying an asymmetric second difference, (c) mask images obtained after morphological processing, and (d) defective, diseased, and contaminated parts of the images after applying masking and thresholding. 
minated apple surfaces showed higher reflectance in the VIS $(>600 \mathrm{~nm})$ and NIR regions compared to the defective or contaminated surfaces, except for bruise spots, which had higher reflectance. Areas with scabs exhibited the lowest reflectance. There was a very distinct absorption feature in the red region of the spectrum with maximum absorption centered at $680 \mathrm{~nm}$. This absorption was due to the presence of chlorophyll $a$ molecules (Chappelle et al., 1992). The contaminated spots lacked the chlorophyll $a$ absorption features, except for bruised areas. Low reflectance characteristics observed from approximately 450 to $550 \mathrm{~nm}$ region for uncontaminated apples were the manifestation of strong absorption by the constituent pigments such as chlorophyll $b$ and carotenoids.

Differentiation between contaminated and defective apples from uncontaminated apples was achieved with multiple wavelength images. Due to the non-flat shape of apples, great differences in reflectance measurements vary across the apples from the centers to the edges. This variation masks the difference that might be seen for either condition. Second difference techniques would allow better differentiation of the contaminated and defective portions of apples. The algebraic expression for the second central difference is given by the following equation:

$$
S^{\prime \prime}\left(\lambda_{\mathrm{n}}, g\right)=S\left(\lambda_{\mathrm{n}}+g\right)-2 S\left(\lambda_{\mathrm{n}}\right)+S\left(\lambda_{\mathrm{n}}-\mathrm{g}\right)
$$

where $S\left(\lambda_{\mathrm{n}}\right)$ is the reflectance image at the center wavelength $\lambda_{\mathrm{n}}$ and $S^{\prime \prime}\left(\lambda_{\mathrm{n}}, g\right)$ is the second difference image at the wavelength $\lambda_{\mathrm{n}}$ with a gap $(g)$ in $\mathrm{nm}$. The center wavelength and the gap were chosen to provide the best contrast between surface defects and uncontaminated portions of the apples. In general, when center spectral

(a)

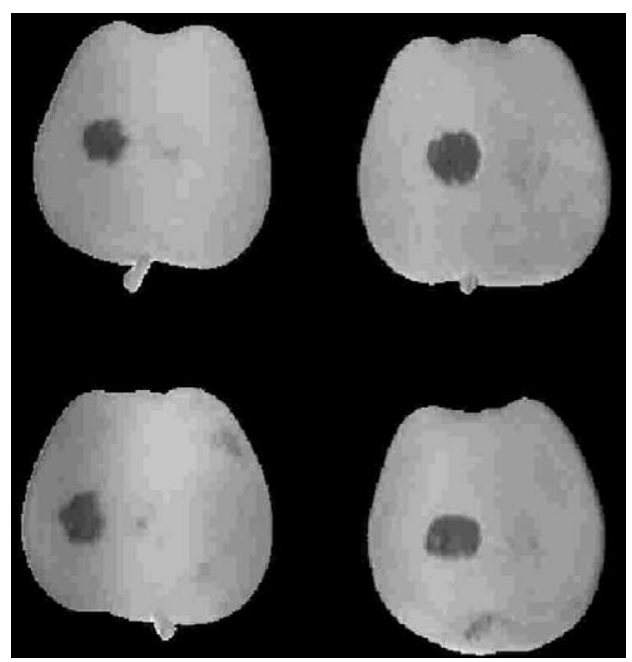

(b)

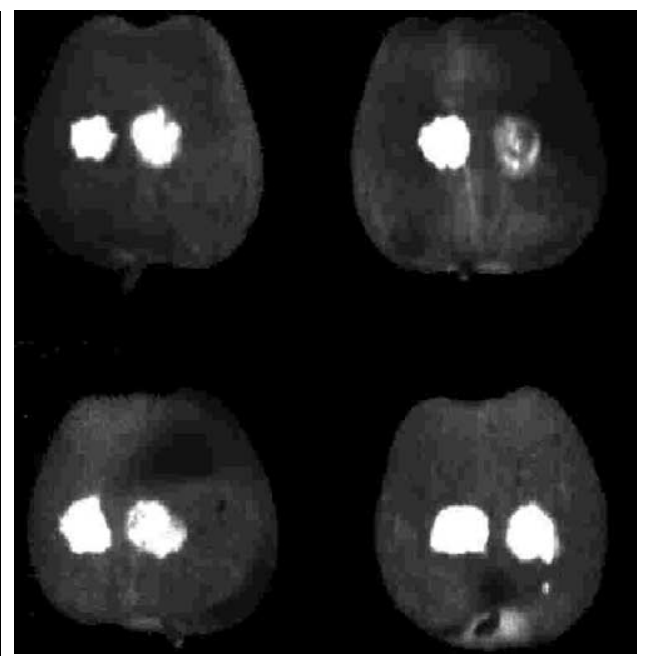

Fig. 10. Ratio images of Red Delicious treated with thick cow manure patches on the left halves and transparent manure spot on the right halves of apples. (a) Reflectance ratio image, R800/R750. (b). Fluorescence ratio image, F680/F450. 
bands are associated with strong pigment absorption features, e.g. carotenoids and chlorophyll $a$, the second difference images provide enhanced visual contrasts between the contaminated and uncontaminated parts of apples as compared to a single waveband image. Fig. 9a shows the use of an absorption feature prominent only to uncontaminated apples, such as the chlorophyll absorption band in the red at $685 \mathrm{~nm}$, as the center band for the second differences method.

To generalize the second difference method with a fixed gap, a modified second difference method, with different gaps (asymmetric) for the lower and upper wavelengths from the center wavelength, was proposed by Mehl et al. (2002):

$$
S^{\prime \prime}\left(\lambda_{\mathrm{n}}, g\right)=S\left(\lambda_{\mathrm{n}}+g_{1}\right)-2 S\left(\lambda_{\mathrm{n}}\right)+S\left(\lambda_{\mathrm{n}}-g_{2}\right)
$$

where $S^{\prime \prime}\left(\lambda_{\mathrm{n}}, g\right)$ is the asymmetric second difference image of $S\left(\lambda_{\mathrm{n}}\right)$ with gaps, $g_{1}$ and $g_{2}$, where $g_{1}$ is not equal to $g_{2}$.

The chlorophyll absorption band centered at $685 \mathrm{~nm}$ with 2 longer wavelengths at 722 and $870 \mathrm{~nm}$, respectively, was found to be very effective in differentiating the contaminated spots from uncontaminated portions of apples. Fig. 9b shows asymmetric second difference images with three bands centered at 685, 720, and $870 \mathrm{~nm}$. Various white spots within the apples depict the defects and contamination on apples. Note that stems were not depicted as defects but as being parts of the uncontaminated apples.

Mask images created with a NIR band and the results of a simple masking and thresholding are also shown in Fig. 9c and d, respectively. All the uncontaminated apples showed no defects or contamination except the one apple positioned at the upper-right corner in Fig. 9d. The white spots shown in Fig. 9d for the uncontaminated apple image is believed to be an actual tiny bruised spot.

Other apple cultivars including Gala, Fuji, and Golden Delicious were also investigated and similar results were obtained. The above three spectral bands can be implemented in a three-channel common aperture imaging system for on-line inspection of apple cultivars for diseases, defects, and soil contamination.

Hyperspectral imaging techniques to develop simple detection methods for fecal contamination on apples were studied (Kim et al., 2000), with both reflectance and fluorescence of fecal contaminated Red Delicious, Gala, Fuji, and Golden Delicious apples. The samples were treated with thick patches of cow manure on the left halves of apples and thin smears (transparent) on the right halves of apples.

Preliminary results showed that a simple ratio between two reflectance images at 750 and $800 \mathrm{~nm}$ bands followed by a simple threshold could differentiate thick patches of manure from regions of uncontaminated surfaces (Fig. 10a). However, for the detection of thin manure spots, multispectral reflectance imaging techniques were not as successful. Further study showed that a simple image ratio of two fluorescence bands at 680 and $450 \mathrm{~nm}$ (Fig. 10b) could easily differentiate uncontaminated portions of apple surfaces from contaminated spots, regardless of apple skin color and thickness of manure treatments. 


\section{Summary}

Machine vision technology has the potential to become very important to the agricultural industry. The use of machine vision technology for land-based and aerial-based remote sensing for natural resources assessments, precision farming, postharvest product quality and safety detection, classification and sorting, and process automation may become routine operations in the near future.

Advances in machine vision technology will make vision systems accurate, robust, and low cost. A real-time operational requirement can be met with a high-speed computer and a frame grabber. The image acquisition board receives imaging data from a camera, performs some processing, and stores the image. It can communicate with the host computer at a speed of 132 Mbytes/second over the PCI bus. These speeds and the data transfer rate are fast enough to meet the real-time needs generally encountered in agricultural applications.

For rapid prototyping of a machine vision system, artificial intelligence programming can be incorporated into the system. Newer tools such as neural networks, fuzzy logic, and expert systems can be applied. For example, Chao et al. (1999) used a color imaging system to classify viscera of wholesome and unwholesome carcasses. They developed a neuro-fuzzy software to enhance the robustness of the classification of the color imaging system.

In order to fully apply machine vision technology, the vision systems for agricultural applications will take full advantage of the fact that vegetation, foods, and agricultural products are biological materials; therefore, the differences in the characteristics of light absorption of the agricultural materials are very important. A hyperspectral imaging technique combines the advantages of spectroscopy and imaging. This technology should find many potential applications in the agricultural industry.

When analyzing hyperspectral image data, the spectral characteristics at each pixel and differences between pixels can be utilized. For example, with hyperspectral imaging of fruits, the specific absorption peaks at chlorophyll and carotenoid bands can be used as a means for the determination of defects, damage, or contamination on the surfaces of fruits. While hyperspectral imaging systems provide important spectral information, they suffer from the incapacity for rapid on-line acquisitions. A common aperture multispectral imaging system with a limited number of wavebands can meet the needs of real-time acquisition and processing. Hyperspectral imaging systems can be used to find optimal bands and develop algorithms for many food commodities. With defined optimal bands, they can be implemented in a common aperture multispectral imaging system for on-line or real-time applications.

\section{References}

Chao, K., Chen, Y.R., Early, H., Park, B., 1999. Color image classification systems for poultry viscera inspection. Appl. Eng. Agric. 15 (4), 363-369. 
Chao, K., Park, B., Chen, Y.R., Hruschka, W.R., Wheaton, F.W., 2000. Design of a dual-camera system for poultry carcasses inspection. Appl. Eng. Agric. 16 (5), 581-587.

Chao, K., Chen, Y.R., Hruschka, W.R., Park, B., 2001. Chicken heart disease characterization by multispectral imaging. Appl. Eng. Agric. 17 (1), 99-106.

Chappelle, E.W., Kim, M.S., McMurtrey, J.E., 1992. Ratio analysis of reflectance spectra (RARS): An algorithm for the remote estimation of the concentrations of chlorophyll a, chlorophyll b, and carotenoids in soybean leaves. Remote Sensing Environ. 39, 239-247.

Chen, P., McCarthy, M.J., Kauten, R., 1989. NMR for internal quality evaluation of fruits and vegetables. Trans. ASAE 32, 1747-1753.

Daley, W., Carey, R., Thompson, C., 1993. Poultry grading/inspection using color imaging. Proc. SPIE 1907, 124-132.

Daley, W., Carey, R., Thompson, C., 1995. Real-time color grading and defect detection of food products. Proc. SPIE 2345, 403-411.

Davenel, A., Guizard, C., Labarre, T., Sevila, F., 1988. Automatic detection of surface defects on fruit using a vision system. J. Agric. Eng. Res. 41, 1-9.

Hwang, H., Park, B., Nguyen, M., Chen, Y.R., 1997. Hybrid image processing for robust extraction of lean tissue on beef cut surfaces. Computers Electronics Agric. 17, 281-294.

Kim, M.S., Chao, K., Chen, Y.R., Chan, D., Mehl, P.M., 2000. Hyperspectral imaging system for food safety: Detection of fecal contamination on apples. In: Chen, Y.R., Tu, S.I. (Eds.), Photonic Detection and Intervention Technologies for Safe Food, Proceedings of SPIE, vol. 4206, pp. 174-184.

Kim, M.S., McMurtrey, J.E., Mulchi, C.L., Daughtry, C.S.T., Chappelle, E.W., Chen, Y.R., 2001 a. Steady-state multispectral fluorescence imaging system for plant leaves. Appl. Optics 40 (1), 157-166.

Kim, M.S., Chen, Y.R., Mehl, P.M., 2001b. Hyperspectral reflectance and fluorescence imaging system for food quality and safety. Trans. ASAE 44 (3), 721-729.

Lu, R., Chen, Y.R., 1998. Hyperspectral imaging for safety inspection of foods and agricultural products. Proc. SPIE 3544, 121-133.

Mao, C., Heitschmidt, J., 1999. Hyperspectral imaging with liquid-crystal tunable filter for biological and agricultural assessment. In: Meyer, G.E., DeShazer, J.A. (Eds.), Precision Agriculture and Biological Quality, Proc. SPIE, vol. 3543, pp. 172-181.

Marks, J.S., Schmidt, J., Morgan, M.T., Nyenhuis, J.A., Stroshine, R.L., 1998. Nuclear magnetic resonance for poultry meat fat analysis and bone chip detection. Industry summary for US Poultry and Egg Association.

Martinsen, P., Shaare, P., 1998. Measuring soluble solids distribution in kiwifruit using near-infrared imaging spectroscopy. Postharvest Biol. Technol. 14, 271-281.

McDonald, T.P., Chen, Y.R., 1991. Visual characterization of marbling in beef ribeyes and its relationship to taste parameters. Trans. ASAE 34 (6), 2499-2504.

McDonald, T.P., Chen, Y.R., 1992. A geometric model of marbling in beef longissimus dorsi. Trans. ASAE 35 (3), 1057-1062.

Mehl, P.M., Chen Y.R., Kim, M.S., 2002. Development of detection methods for defects and contaminations on apple surface using hyperspectral imaging technique, J. of Food Engineering, in review.

Muir, A.Y., Porteous, R.L., Wastie, R.L., 1982. Experiments in the detection of incipient diseases in potato tubers by optical methods. J. Agric. Eng. Res. 27, 131-138.

Park, B., Chen, Y.R., 1994. Intensified multispectral imaging system for poultry carcass inspection. Trans. ASAE 37 (6), 1983-1988.

Park, B., Chen, Y.R., 1996. Multispectral image co-occurrence matrix analysis for poultry carcasses inspection. Trans. ASAE 39 (4), 1485-1491.

Park, B., Chen, Y.R., Chao, K., 1998. Multispectral imaging for detecting contamination in poultry carcasses. In: Chen, Y.R. (Ed.), Pathogen Detection and Remediation for Safe Eating, Proceedings of SPIE, vol. 3544, pp. 156-165.

Rehkugler, G.E., Throop, J.A., 1989. Image processing algorithm for apple defect detection. Trans. ASAE $32,267-272$.

Rigney, M.P., Brusewitz, G.H., Stone, M.L., 1996. Peach physical characteristics for orientation. Trans. ASAE 39, 1493-1497. 
Rumbaugh, J., Blaha, M., Premerlani, W., Eddy, F., Lorensen, W., 1991. Object-Oriented Modeling and Design (chapter 19). Prentice-Hall, Englewood Cliffs, New Jersey, pp. 417-432.

Schatzki, T.F., Haff, R.P., Young, R., Can, I., Le, L.C., Toyofuku, N., 1997. Defect detection in apples by means of X-ray imaging. Trans. ASAE 40, 1407-1415.

Singh, N., Delwiche, M.J., 1994. Machine vision methods for defect sorting stonefruit. Trans. ASAE 37, 1989-1997.

Taylor, S.K., McCure, W.F., 1989. NIR imaging spectroscopy: Measuring the distribution of chemical components. In: Proceedings of the Second International NIR Conference, Tsukuba, Japan pp. $393-$ 404.

Throop, J.A., Aneshansley, D.J., Upchurch, B.L., 1993. Near-IR and color imaging for bruise detection on Golden Delicious apples. Proc. SPIE 1836, 33-44.

Throop, J.A., Aneshansley, D.J., Upchurch, B.L., 1995. An image processing algorithm to find new and old bruises. Appl. Eng. Agric. 11, 751-757. 